\title{
PREVALÊNCIA DE BURNOUT EM ENFERMEIROS DO HOSPITAL METROPOLITANO DE URGÊNCIA E EMERGÊNCIA, POR MEIO DO QUESTIONÁRIO MASLACH
}

Tereza Cristina Dos Reis FERREIRA ${ }^{1}$

João Felipe França Calandrini de AZEVEDO²

Lorrane Rocha da CUNHA ${ }^{3}$

Aline Caldas da CUNHA ${ }^{4}$

Nayara Emilia Seabra Oliveira CARDOSO ${ }^{5}$

${ }^{1}$ UEPA - Departamento do Movimento Humano-Curso de Fisioterapia. CESUPA -Areas das Ciências Ambientais,B iológicas e da Saúde- Curso de Fisioterapia.

tereza_reis@yahoo.com.br

${ }^{2}$ CESUPA -Áreas das Ciências Ambientais, Biológicas e da Saúde- Curso de Fisioterapia. felipecalandrini@yahoo.com.br

${ }^{3}$ CESUPA -Áreas das Ciências Ambientais, Biológicas e da Saúde- Curso de Fisioterapia. lorrane_rocha92@hotmail.com

${ }^{4}$ CESUPA -Áreas das Ciências Ambientais, Biológicas e da Saúde- Curso de Fisioterapia. alinefices@yahoo.com.br.

${ }^{5}$ CESUPA -Áreas das Ciências Ambientais, Biológicas e da Saúde- Curso de Fisioterapia.

naya.o.cardoso@hotmail.com

Recebido em: 07/12/2014 - Aprovado em: 03/06/2015 - Disponibilizado em: 15/07/2015

\begin{abstract}
RESUMO
O estresse relacionado ao trabalho ou estresse ocupacional, vem ganhando destaque na comunidade cientifica devido sua elevada capacidade em promover alterações na saúde mental e comportamental do trabalhador. A equipe de enfermagem tem como características da profissão uma elevada carga de trabalho, que além do contato com os pacientes e familiares, também existe a função de preencher protocolos, evoluções, encaminhamentos e toda uma burocracia a ser somada nas atividades laborais da instituição. A síndrome de Burnout quando ao acometer o indivíduo, promove somente reações negativas no cotidiano deste, chegando a evitar o bom relacionamento com os demais colegas de profissão e contribuindo negativamente para a instituição que o emprega. O objetivo deste estudo foi verificar a prevalência da síndrome de Burnout em enfermeiros do hospital metropolitano de urgência e emergência (HMUE), por meio do questionário Maslach. Foram entrevistados para a pesquisa 51 enfermeiros de ambos os sexos do (HMUE) onde as informações foram obtidas por meio do questionário Maslach (Maslch Burnout Inventory) - composta por 22 afirmativas relacionadas a sentimentos e atitudes que envolvem os três aspectos fundamentais da síndrome, divididos em três escalas de sete pontos, que variam de 0 a 6 . Desta forma, os enfermeiros do HMUE apresentam um alto nível de estresse com predisposição para manifestação da síndrome de Burnout, com nível alto para exaustão emocional, nível moderado para despersonalização e nível baixo para realização pessoal.
\end{abstract}

Palavras -chave : Esgotamento profissional. Enfermagem. Despersonalização. Hospitais. Questionários. 


\title{
BURNOUT PREVALENCE IN NURSES OF METROPOLITAN URGENCY AND EMERGENCY HOSPITAL THROUGH MASLACH QUESTIONARY
}

\begin{abstract}
The work-related stress, occupational stress, is gaining prominence in the scientific community because of its high capacity to promote changes in behavioral and mental health of the worker. The nursing team has the characteristics of the profession a high workload, which in addition to contact with the patient and family, there is also the fill function protocols, developments, referrals and a whole bureaucracy to be added in the labor activities of the institution. Burnout syndrome when to involve the individual, only promotes negative reactions in this everyday, getting to avoid the good relationship with other professional colleagues and contributing negatively to the institution that employs him. The objective of this study has determined the prevalence of burnout in nurses of the Metropolitan Hospital Urgency and Emergency syndrome (HMUE), through the Maslach questionnaire. Were interviewed for the survey 51 nurses of both sexes of (HMUE) where the information was obtained through the Maslach questionnaire (Maslach Burnout Inventory) - composed of 22 questions related to feelings and attitudes involving the three fundamental aspects of the syndrome, divided three seven-point scale, ranging from 0 to 6 . This, HMUE nurses have a high level of stress with predisposition for the manifestation of burnout, with a high level for emotional exhaustion, depersonalization and moderate level to low level for personal fulfillment.
\end{abstract}

Keywords: Bornout, Professional. Nursing. Despersonalization. Hospitals. Questionnaires.

\section{INTRODUÇÃO}

Antes do termo técnico, Síndrome de Burnout, a patologia passou por outras nomenclaturas, como a Síndrome do Assistente Desassistido, ou então a Síndrome do Cuidador Descuidado, nos dois casos, o nome revela a falta de atenção que o profissional tem com ele. A metáfora da brasa que virou cinza é usada por psicólogos com seus pacientes para que de uma maneira figurada eles consigam identificar $o$ sentimento que possuem ao pensar no trabalho (SANTOS, 2009).

O Termo Burnout foi divulgado pela primeira vez na comunidade, pelo médico Herbert Freundenberger em uma revista de psicologia no ano de 1974, porém só em 1977, no Congresso Anual da Associação
Americana de Psicólogos que o termo se tornou popular pelos psicólogos, Maslach e Pines (BEZERRA; BERESIN, 2009).

O avanço no estudo sobre estresse evidenciou a síndrome de Burnout, que é caracterizada no indivíduo, quando existe a perda de sentido do labor, e todo o esforço necessário para realizá-lo, passa a ser um fardo desnecessário para esse trabalhador (FRANÇA, 2010).

Para Maslach et al (1982 apud BARROS et al, 2008), Burnout é uma síndrome de esgotamento profissional, proveniente da exposição prolongada a fatores interpessoais crônicos no trabalho e apresentando três dimensões: exaustão emocional, despersonalização e ineficácia. 
A exaustão emocional trata-se do momento em que não há mais força quando se pensa que no dia seguinte existe mais uma jornada de trabalho, é quando toda a sua energia foi consumida por todos aqueles que foram assistidos por você, é quando o ambiente de trabalho consegue te absorver por inteiro. A despersonalização é entendida quando existe a mudança nas atitudes de assistência, é quando o sentimento de cuidado é substituído por sentimentos e atitudes negativas, quando o cinismo toma conta e o zelo pelo assistido deixa de existir, tornando o indivíduo "desumano" (MASLACH, 1982). A ineficácia fica evidenciada quando há a falta de interesse nas atividades laborais, onde o indivíduo vai perdendo o interesse, e surge um sentimento de descontentamento. É quando o labor perde o sentido e passa a ser um fardo (BEZERRA; BERESIN, 2009).

A síndrome de Burnout tem preferência por trabalhadores que mantém o contato direto com outras pessoas no nível do cuidado. $\mathrm{O}$ cuidador tem uma íntima relação com os familiares e o paciente enfermo, a constante proximidade com o sofrimento. Dor e a morte, a desvalorização do profissional pelo próprio profissional, a sobrecarga de trabalho, a escassez de equipamentos para uma melhor realização do trabalho, o medo de criticas pelo baixo desempenho laboral, são fatores que determinam o surgimento desta patologia (SANTOS, 2009).
A síndrome não é restrita a profissionais que tratam do cuidado, ela vem sendo estudada também fora do âmbito profissional, como por exemplo, atividades relacionadas ao lar, às donas de casa. Conforme a Health Education Authority, a enfermagem está classificada como a quarta mais estressante do setor público (MOREIRA et al, 2009).

No cotidiano hospitalar, podemos afirmar que a equipe de enfermagem, compreendida entre enfermeiros, técnicos e auxiliares, são os que mais mantêm a proximidade com o paciente e seus familiares, devido o foco da profissão ser constituído de cuidado. Essa categoria no ambiente de trabalho, é a mais predisposta ao desenvolvimento da síndrome de Burnout por ter como fardo um significativo contato com o sofrimento alheio (MOREIRA et al, 2009).

A equipe de enfermagem tem como características da profissão uma elevada carga de trabalho, que além do contato com os pacientes e familiares, também existe a função de preencher protocolos, evoluções, encaminhamentos e toda uma burocracia a ser somada nas atividades laborais da instituição. Toda essa atividade predispõe a equipe ao evento do estresse laboral (MENEGHINI, PAZ, LAUTERT, 2011).

A enfermagem tem também como fardo a tomada de decisões e procedimentos para o tratamento, o que pode interferir 
diretamente na melhora ou piora do paciente, o que não costuma acontecer em outras profissões, esse agente estressor é típico da categoria. $\mathrm{O}$ estresse quando instalado no profissional de enfermagem, chega a interferir, proporcionando tensão sobre aquele que é o alvo do trabalho, o cliente, no caso da enfermagem, o paciente (GOMES, CRUZ, CABANELAS, 2009).

A sobrecarga, tanto na qualidade quanto na quantidade de trabalho, como a deficiência na comunicação com os companheiros, o cotidiano de sofrimento e morte, o fluxo emocional é estressante, a constante permutação no emprego, a ausência de continuidade com o paciente, o conflito hierárquico e de autoridades e a falta de autonomia, por depender da aprovação de outros profissionais, são enclaves da prática da profissão que proporcionam o estresse (LAUTERT, 1995).

As fases da síndrome de Burnout na equipe de enfermagem, afeta direta e negativamente o principal alvo do cuidado. $\mathrm{O}$ desgaste emocional e profissional que os enfermeiros sofrem devido à tensão emocional crônica, mostra que eles trabalham de mais, o que dificulta as relações e o enfrentamento de situações clinicas graves, pois existe a manifestação de insatisfação e de perda de comprometimento, percebendo que de fato há menos tempo para eles, levando de forma subsequente às demais fases da síndrome, que são a despersonalização e ineficácia (TAMAYO; TRÓCCOLI, 2002; JODAS; HADDAD 2009).

Até o presente momento, não foi elucidada na literatura nenhum trabalho na região Norte e em Belém, que trate de forma generalizada os enfermeiros com a predisposição à síndrome de Burnout. Diante do exposto o objetivo geral deste estudo foi verificar a prevalência de Burnout em Enfermeiros do Hospital Metropolitano de Urgência e Emergência, por meio do questionário Maslach.

\section{METODOLOGIA}

Todos os indivíduos/sujeitos da presente pesquisa foram estudados segundo as Normas de Pesquisa Envolvendo Seres Humanos (Res. CNS 466/12) do Conselho Nacional de Saúde, após a submissão e aprovação do projeto pelo Comitê de Ética em Pesquisa do Centro Universitário do Pará sob o número CAAE 15608613.7.0000.5169, após o aceite da orientadora, após o aceite do Hospital Metropolitano de Urgência e Emergência, e pelos indivíduos por meio do Termo de Consentimento Livre e Esclarecido (TCLE). A pesquisa foi do tipo observacional, prospectivo. A pesquisa foi realizada com 51 enfermeiros do Hospital Metropolitano de Urgência e Emergência. Esta pesquisa teve como critérios de inclusão, profissionais graduados em enfermagem de ambos os sexos, que realizem trabalhos em ambientes 
hospitalares. Técnicos de enfermagem, Enfermeiros de licença ou de férias. As informações foram obtidas por meio do Questionário Maslach (Maslach Burnout Inventory) - composto por vinte e duas afirmações sobre sentimentos e atitudes que englobam os três aspectos fundamentais da síndrome, divididos em três escalas de sete pontos, que variam de 0 a 6. Desta forma, foram descritas, de forma independente, cada uma das dimensões que caracterizam a estafa profissional. A exaustão emocional foi avaliada por nove itens, a despersonalização por cinco e a realização pessoal por oito itens. As notas de corte utilizadas foram às empregadas no estudo de Maslach. Para exaustão emocional, uma pontuação maior ou igual a 27 indica alto nível, de 19 a 26 nível moderado e abaixo de 19 nível baixo. Para despersonalização, pontuações iguais ou maiores a 10 indicam alto nível, de 06 a 09 nível moderado e menores de 06 nível baixo. A pontuação relacionada à realização pessoal vai em direção oposta às outras, uma vez que pontuações de zero a 33 indicam alto nível, de 34 a 39, nível moderado e maior ou igual a 40, nível baixo. Sendo que altos níveis de exaustão emocional e de despersonalização e nível baixo de realização pessoal caracterizam Burnout. Após a coleta de dados foi elaborado uma planilha eletrônica, para armazenamento dos dados, no software Microsoft Excel ${ }^{\circledR}$ 2010, na qual cada linha corresponde a um caso e cada coluna a uma variável. Para representação descritiva dos dados foram elaboradas tabelas, utilizando o Software Microsoft Word®, representando as médias e desvio-padrão das distribuições das variáveis numéricas. A análise dos dados foi processada utilizando-se o programa SPSS Statistics 17.0.

\section{RESULTADOS}

A casuística inicial para este estudo era de 90 enfermeiros e teve como casuística final 51 enfermeiros de todos os setores do Hospital Metropolitano de Urgência e Emergência, unidades de terapia intensiva, enfermarias e pronto atendimento, as perdas ocorrerão em virtude de enfermeiros em férias, licença e afastamentos do trabalho.

A seguir serão apresentados os dados colhidos por meio do Maslach Burnout Inventory, e no estudo, foram analisados os seguintes itens: Exaustão emocional, despersonalização e realização profissional.

Em relação às dimensões analisadas nos enfermeiros do Hospital Metropolitano de Urgência e Emergência, obtiveram-se os seguintes dados: na dimensão exaustão emocional a média foi 30,5 no valor do escore dos enfermeiros entrevistados, mostra que ha um alto nível para a exaustão emocional; em relação a despersonalização a média de 8,4 no valor do escore dos enfermeiros entrevistados, mostra um nível moderado para despersonalização. Em relação à realização 
profissional a média foi de 40,7 no valor do escore dos enfermeiros entrevistados, mostra um nível baixo para a realização profissional, sendo os dados sugestivos de Burnout nos enfermeiros do HMUE (TABELA1).

Tabela 1. Média e desvio-padrão das dimensões do questionário Maslach dos enfermeiros do Hospital Metropolitano de Urgência e Emergência

\begin{tabular}{lcc}
\hline Dimensões & $\begin{array}{l}\text { Média } \\
(\mathbf{n = 5 1})\end{array}$ & $\begin{array}{c}\text { Desvio- } \\
\text { padrão }\end{array}$ \\
\hline Exaustão emocional & 30,5 & \pm 11 \\
Despersonalização & 8,4 & \pm 6 \\
Realização Pessoal & 40,7 & $\pm 5,6$ \\
\hline
\end{tabular}

Fonte: Pesquisa de campo, 2013.

\section{DISCUSSÃO}

A síndrome de burnout é caracterizada pelo esgotamento físico, psíquico e emocional, em decorrência de trabalho estressante e excessivo. É um quadro clínico resultante da má adaptação do homem ao seu trabalho. É compreendida como uma reação de estresse crônico e se caracteriza por reações como exaustão emocional e física, perda de sentimento de realização no trabalho com produtividade diminuída, despersonalização extrema com respeito às outras pessoas, manifestando-se através de atitudes negativas para com as pessoas no trabalho, sendo, portanto uma experiência pessoal de esgotamento (CARTOLLO, 2002).

Apesar de qualquer pessoa poder vir a sofrer de estresse ocupacional em função das atividades desenvolvidas, o burnout incide principalmente nos que ajudam, prestam assistência ou são responsáveis pelo desenvolvimento de outros, tais como: médicos, enfermeiros, professores, assistentes sociais, psicólogos, dentistas, bombeiros, agentes penitenciários, policiais, enfim, cuidadores em geral (ABREU et al, 2002; CARTOLLO, 2002; TUCUNDUVA et al, 2006). O processo do burnout é individual, sua evolução leva anos e até mesmo décadas. Seu surgimento é paulatino, cumulativo, com incremento progressivo em severidade, não sendo percebido pelo individuo, que geralmente se recusa a acreditar estar acontecendo algo de errado com ele (CARTOLLO, 2002).

O burnout é definido como uma síndrome psicológica decorrente da tensão emocional crônica no trabalho. Trata-se de uma experiência subjetiva interna que gera sentimentos e atitudes negativas no relacionamento do individuo com o seu trabalho (insatisfação, desgaste, perda de comprometimento), minando o seu desempenho profissional e trazendo conseqüências indesejáveis para a organização (absenteísmo, abandono de emprego, baixa produtividade). É caracterizado pelas dimensões; exaustão emocional, que refere à sensação de esgotamento tanto física como mental, levando o indivíduo a se sentir sem energia para absolutamente nada. Esse sentimento pode se desenvolver pelo envolvimento 
emocional em demasia e pelo fato do profissional ter que se confrontar com pessoas que padecem necessidades, problemas e sofrimentos. Despersonalização onde, para evitar o esgotamento emocional, o indivíduo reduz seu contato com pessoas ao mínimo, distanciando-se, evitando envolver-se emocionalmente, esquivando-se do trato individual, mantendo relações frias e distantes com os pacientes (STACCIARINI, 1999).

Segundo Galvão, Sawada e Trevizan (2004) a profissão de enfermagem surgiu a partir de evidencias de teorias e pesquisas para tomadas de decisão com relação ao cuidado, prestado a um grupo de pacientes, levando em conta as necessidades individuais e preferencias de cada um.

No Brasil, pode-se considerar que o ensino oficial sistematizado da enfermagem moderna foi introduzido a partir de 1923 pelo decreto de lei $n^{\circ}$ 16300/23 no Rio de Janeiro, mediante a organização do serviço de enfermeiras do Departamento Nacional de Saúde Pública (DNSP) que posteriormente foi denominada escola de enfermagem Anna Nery, segundo Ito, Peres, Takahashi, Leite (2006).

Segundo Gomes e Oliveira (2005), a formação dos profissionais de enfermagem sofre com a disciplinarização de seus alunos, tornando-os subordinados, apesar da evolução da área, e no crescimento da enfermagem tanto no âmbito teórico, quanto no prático, pois o mercado acaba exigindo o profissional que tenha agilidade e rapidez, do que valorizar a capacidade do pensar como prioridade no cotidiano de trabalho.

Netto e Ramos (2002), o cotidiano de trabalho da enfermagem, torna-se conflituoso devido o enfermeiro ser mediador de desentendimentos da equipe médica, e também muitas vezes os conflitos são entre médicos e enfermeiros, tendo em vista que a equipe de enfermagem é sempre a culpada por procedimentos realizados sem sucesso.

Stacciarini e Troccoli (2001),a natureza e a característica do trabalho do enfermeiro, predispõe a manifestação do fenômeno do estresse, devido a rotineira presença de agentes estressores no cotidiano de trabalho, pois desde o surgimento da profissão, o enfermeiro busca construir sua identidade profissional e obter o reconhecimento devido.

Este estudo não teve como objetivo avaliar o número de empregos dos enfermeiros, mas sim, de analisar se a atuação dos enfermeiros em um hospital de alta e média complexidade interfere no estresse da jornada. Sendo evidenciada na dimensão exaustão emocional a média de 30,5 no valor do escore dos enfermeiros entrevistados, mostrando que há um alto nível para a exaustão emocional; em relação a despersonalização a média de 8,4 no valor do escore dos enfermeiros entrevistados, mostra 
um nível moderado para despersonalização. Em relação à realização profissional a média foi de 40,7 no valor do escore dos enfermeiros entrevistados, mostra um nível baixo para a realização profissional, sendo os dados sugestivos de Burnout nos enfermeiros do HMUE.

Fascina et al (2007), em seu estudo realizado dentro de uma UTI adulto, os resultados apontam que $65,5 \%$ apresentam um alto índice de exaustão emocional, $10,34 \%$ um alto nível para despersonalização e $17,25 \%$ alto para reduzida realização profissional, logo não pode-se dizer que esta população apresenta alto índice para a síndrome de Burnout, corroborando com os dados deste estudo. Sendo evidenciado também no estudo de Jodas e Haddad (2009), onde o grupo formado por 61 profissionais de enfermagem de um hospital universitário, verificou-se que $21,3 \%$ dor profissionais apresentam alta classificação para a exaustão emocional, 32,8\% alta classificação para a despersonalização e $26,2 \%$ apresenta baixa classificação para realização profissional, características estas que estabelecem diagnóstico para a manifestação da síndrome de Burnout.

Descordando com os achados neste estudo evidenciou- se o estudo de Moreira et al (2009), em seu estudo no hospital Nossa Senhora da Conceição, no sul do Brasil, através do questionário Maslach Burnout
Inventory (MBI), os valores médios obtidos nas três dimensões da síndrome de Burnout foram 17 pontos em cansaço emocional, indicando nível baixo; 7,79 pontos em despersonalização, indicando nível médio; e 36,6 pontos em realização pessoal, nível médio.

Bezerra e Beresin (2009) no grupo de 17 enfermeiros de uma equipe de regaste préhospitalar verificou-se que a maioria da amostra um total de $74,47 \%$ apresentou baixo/moderado para os níveis de exaustão emocional, despersonalização e realização profissional.

Galindo et al (2012) em seu estudo sobre Síndrome de Burnout entre enfermeiros de um hospital geral da cidade do Recife, identificou em 64 enfermeiros que 50\% dos profissionais apresentava altos níveis de exaustão emocional, 27,0\% despersonalização e 4,8\% revelaram alto nível de realização pessoal no trabalho, não caracterizando dados sugestivos de Burnout.

A enfermagem é considerada uma das profissões mais estressantes dentro do hospital. Em um Hospital de referência em trauma e queimaduras em especial possui uma gama de atividades de média e alta complexidade que requer atenção, competência e dedicação constante do profissional.

É importante relacionar as fontes de tensão no trabalho para que, através delas, se 
possa intervir fazendo a prevenção (sistemas de trabalho, horários, tempo livre, atividades extra-trabalho, incentivos, salários, etc).

Ao pensar em um plano de prevenção para evitar a ocorrência da síndrome de burnout é necessário que este seja individualizado, verificando através de pesquisa interna o que cada profissional sente no seu ambiente de trabalho e o que o mesmo relaciona como estressante e desgastante ou ainda despersonalizante, assim como ele percebe sua profissão e como se percebe nela.

Alguns pontos destacados a seguir merecem atenção especial, pois oferecem algumas possibilidades de melhoria para o profissional em seu ambiente de trabalho, uma convivência diária mais positiva e a possibilidade de realização profissional e pessoal.

O trabalho em equipe pode ajudar a conhecer-se e compartilhar as responsabilidades; a realização de cursos de aperfeiçoamento ajuda a incorporar conhecimentos e realizar uma pausa nas atividades diárias; atividade física e de recreação ao longo do trabalho ajudam a descontrair, assim como um espaço de reflexão sobre as tarefas diárias com os demais profissionais, poderia permitir a possibilidade de clarificação das situações vivenciadas; o cultivo da solidariedade e do humor pode ajudar a enfrentar o desgaste, bem como o rodízio de setores onde as exigências de trabalho são menores ajudará a sobrecarga.

Associados a estes fatores há outros, como atividade social fora do ambiente de trabalho com o grupo de profissionais da unidade; algumas alterações serem tomadas em conjunto com os profissionais da unidade; confraternização semanal ou mensal pra possibilitar ao grupo se encontrar, se não der pra ser com todos os plantões juntos, que cada plantão faça separado e no dia e horário mais apropriado; que seja oferecida ao profissional a possibilidade de troca de plantão quando o mesmo tiver algum compromisso importante e de última hora, para que o profissional possa ter uma vida social e participar da vida social da família, o que muitas vezes ele deixa de fazer (atividades diversas); deixar que a equipe faça sua própria escala mensal de folga, dando a possibilidade de adequar à sua necessidade e ao seu lazer, isso tudo dentro de regras para que não desfalque o setor; dar folga extra quando o setor se destacar pelas suas atividades.

A Síndrome de Burnout é uma patologia nova, com muitos fatores que facilitam o seu surgimento e sintomas que muitas vezes os profissionais desconhecem, deste modo não percebe quando estão desenvolvendo-a. Sabemos que essa síndrome hoje, está presente em todas as profissões, sendo necessários mais estudos a seu respeito para entendê-la melhor, possibilitando assim, 
condições de trabalho mais adequadas para que todos os profissionais, quer da área da saúde ou não, sentiam satisfação no trabalho que estão desenvolvem.

\section{CONSIDERAÇÕES FINAIS}

A prevalência da Síndrome de Burnout em enfermeiros do Hospital Metropolitano de Urgência e Emergência do Pará se enquadra no alto nível de exaustão emocional $(30,5)$, despersonalização $(8,4)$ e baixo nível de realização profissional (40,7), com dados sugestivos de alto nível de Burnout.

O estresse já se tornou uma epidemia, e vem crescendo em proporções assustadoras, e a forma com que cada indivíduo absorve as cargas estressantes do dia a dia é de forma individual, e dependendo da pessoa, pode acarretar no desenvolvimento da síndrome de Burnout, causando um esgotamento físico e mental, causando impactos negativos na vida deste profissional.

Existem estudos a respeito da população de enfermeiros relacionados à Síndrome de Burnout, porém há necessidade de estudos contínuos com a categoria, para que haja uma possibilidade destes profissionais em lidar de forma mais clara com os fatores que predispõem ao surgimento desta síndrome, com isso, melhorando a saúde do enfermeiro e aumentando a qualidade da assistência e obtendo um bom relacionamento com os colegas de trabalho.

\section{REFERÊNCIAS}

ABREU, L.A. et al. Estresse ocupacional e Síndrome de Burnout no exercício profissional da psicologia. Psicologia: ciência e profissão. v.22, pag 22-29, 2002.

BARROS, D. et al. Médicos plantonistas de unidade de terapia intensiva: perfil sóciodemográfico, condições de trabalho e fatores associados à síndrome de burnout.

BEZERRA,R; BERESIN, R. A síndrome de burnout em enfermeiros da equipe de resgate pré-hospitalar.

CARLOTTO, M.S. A síndrome de Burnout e o trabalho docente. Rev.Psicologia em estudo. 7(1):21-29, 2002

FASCINA, L.P, et al. Avaliação do nível da Síndrome de Burnout na equipe de enfermagem da UTI-adulto. Prod Cient, Hospital Sepaco, São Paulo, p. 2-28, 2007.

FRANÇA, M. F. Estudo sobre síndrome de burnout em profissionais de enfermagem em dois hospitais de médio porte no município de Cáceres - MT.

GALVÃO, C. M; SAWADA, N. O.;

TREVIZAN, M. A. REVISÃO

SISTEMÁTICA. Rev Latino-am

enfermagem, v. 12, n. 3, p. 549-56, 2004.

GOMEZ, R. A; CRUZ, F. J; CABANELAS, $S$. Estresse ocupacional em profissionais de saúde: um estudo com enfermeiros portugueses.

GOMES, A. M. T; OLIVEIRA, D. C. Estudo da estrutura da representação social da autonomia profissional em enfermagem.

ITO, et al. O ensino de enfermagem e as diretrizes curriculares nacionais: utopia $\mathrm{x}$ realidade. 
JODAS, A. D; HADDAD, L. C. M. Síndrome de Burnout em trabalhadores de enfermagem de um pronto socorro de hospital universitário.

LAUTERT, L. O desgaste profissional do enfermeiro.

MASLACH, C; JACKSON, S.E. A social psychological analysis. Social psychology of health and illness, p. 227, 1982.

MENEGHINI, F; PAZ, A. A; LAUTERT, L. Fatores ocupacionais associados aos componentes da síndrome de burnout em trabalhadores de enfermagem.

MOREIRA, S. D, et al. Prevalência da síndrome de burnout em trabalhadores de enfermagem de um hospital de grande porte da Região Sul do Brasil.

NETTO, L. F. S. A; RAMOS, F. R.

S. Enfermeiro: O papel que se define nas relações conflituosas.

SANTOS, W. J. A síndrome de burnout: Uma análise social e psicodinâmica.

SONNENSCHEIN, S, et al. Parents' beliefs about children's math development and children's participation in math activities. Child Development Research, v. 2012, 2012.

STACCIARINI, J.M.R. Estresse ocupacional, estilos de pensamentos e coping - na satisfação, mal-estar físico e psicológico dos enfermeiros. [Tese]. Inst Psi Soc Trab, Brasília, 1999.

STACCIARINI, J.M.R; TRÓCCOLI, B.T. O estresse na atividade ocupacional do enfermeiro. Rev Latino-am Enferm, v. 9, n. 2, p. 17-25, 2001.
TAMAYO, R. M. TRÓCCOLI, T. B. Exaustão emocional: relações com a percepção de suporte organizacional e com as estratégias de copingno trabalho.

TUCUNDUVA, L.T.C.M, et al. A síndrome da estafa profissional em médicos cancerologistas brasileiros. Rev Assoc. Med. Bras. 5(22):108-112. 2006. 